\title{
A Discrete Element Method for modelling cell mechanics - application to the simulation of chondrocyte behavior in the growth plate
}

\author{
Grand R. Joldes ${ }^{1,2}$, George C. Bourantas ${ }^{2}$, Adam Wittek ${ }^{2}$, Karol Miller, ${ }^{2,3}$, \\ David W. Smith ${ }^{4}$, Bruce S. Gardiner ${ }^{1}$ \\ ${ }^{1}$ School of Engineering and Information Technology, Murdoch University, 90 South St,
Murdoch, Australia
${ }^{2}$ Intelligent Systems for Medicine Laboratory, The University of Western Australia, Perth,
Australia
${ }^{3}$ School of Engineering, Cardiff University, The Parade, CF24 3AA Cardiff, UK
${ }^{4}$ Engineering Computational Biology, School of Computer Science and Software Engineer-
ing, The University of Western Australia, Perth, Australia
}

Abstract

In this paper we describe a discrete element method (DEM) framework we have developed for modelling the mechanical behavior of cells and tissues. By using a particle method we are able to simulate mechanical phenomena involved in tissue cell biomechanics (such as extracellular matrix degradation, secretion, growth) which would be very difficult to simulate using a continuum approach.

We use the DEM framework to study chondrocyte behavior in the growth plate. Chondrocytes have an important role in the growth of long bones. They produce cartilage on one side of the growth plate, which is gradually replaced by bone. We will model some mechanical aspects of the chondrocyte behavior during two stages of this process.

The DEM framework can be extended by including other mechanical and chemical processes (such as cell division or chemical regulation). This will help us gain more insight into the complex phenomena governing bone growth.

Keywords: Discrete Element Method, chondrocyte, extracellular matrix, bone growth, growth plate 


\section{Introduction}

The behavior of cells and tissues in the human body is governed by complex, interconnected physical phenomena. The mechanical behavior of cells is regulated by complicated chemical signaling pathways, with different signals either promoting or inhibiting certain cell activities [1]. Computer modelling can help us understand these complex connections and the effect of different signals on the cell and tissue behavior. A first step in this direction consists of the ability to model the mechanical behavior of cells.

From a mechanical point of view, modelling cell behavior is complicated by the multitude of phenomena and interactions that have to be taken into account, including cell growth and proliferation, secretion, degradation of the extra cellular matrix (ECM), active interaction with the ECM and cell death [1]. Such complex behavior would be very difficult to model using a continuum approach. Therefore, we have proposed to use the discrete element method (DEM), with the domain of interest (cells, ECM) is discretized using simple round particles. The behavior of cells and tissues emerges from simple interaction laws between the domain discretizing particles [2]. This allows the simulation of complex cell behavior with relatively low implementation and computation cost.

We will use the proposed DEM framework to model the behavior of chondrocytes in the epiphyseal growth plate. The chondrocytes are involved in the longitudinal growth of long bones through the endochondral ossification process, in which new cartilage is formed at one side of the epiphyseal growth plate and is gradually replaced by bone. Growth plate chondrocytes differentiate through multiple stages of this process (from a resting state through proliferative, prehypertrophic and hypertrophic stages) as the growth plate moves past. The differentiation pathway ends in cell death and the replacement of the chondrocyte generated cartilage by bone [3]. Chondrocytes synthetize the cartilage extra cellular matrix (ECM), consisting of collagen fibers and non-collagenous glycoproteins, hyaluronan and proteoglycans. The ECM defines the mechanical and physical properties of cartilage $[4,5]$.

In this paper we described the proposed DEM framework and use it to simulate some of the mechanics of chondrocyte behaviour in the growth plate during two of the stages of the bone growth process. In our approach, each cell (and the ECM) is modelled by a collection of particles. This allows us to control the properties and behaviour of different parts of the cell (such as the mechanical properties of cytoplasm and nucleus or the interaction properties between different parts of the cell membrane and the ECM). The simulated phenomena require the modelling of complex behaviour such as cell growth, secretion and degradation of ECM.

The paper is organized as follows: the discrete element model used is described in the next Section, followed by the results of simulating mechanical behavior of chondrocytes in the growth plate and ending with the discussion and conclusions Section. 


\section{Discrete Element Model of the Chondrocyte and ECM}

\section{Domain Discretization and the Equation of Motion}

In our DEM framework the physical domain (in our case a chondrocyte and the surrounding ECM) is discretized using a set of circular particles. The macroscopic behavior of the cell and surrounding tissue is governed by simple interaction laws between neighboring particles (Figure 1). The ECM, cell membrane, cytoplasm, nucleus membrane and nucleus are all discretized using particles having different properties [2]. Furthermore, membrane particles are differentiated based on their position in order to capture the behavior of polarized cells; particles on the leading or trailing edge of the cell can have special behavior which allows the modelling of ECM degradation or hyaluronic acid production.

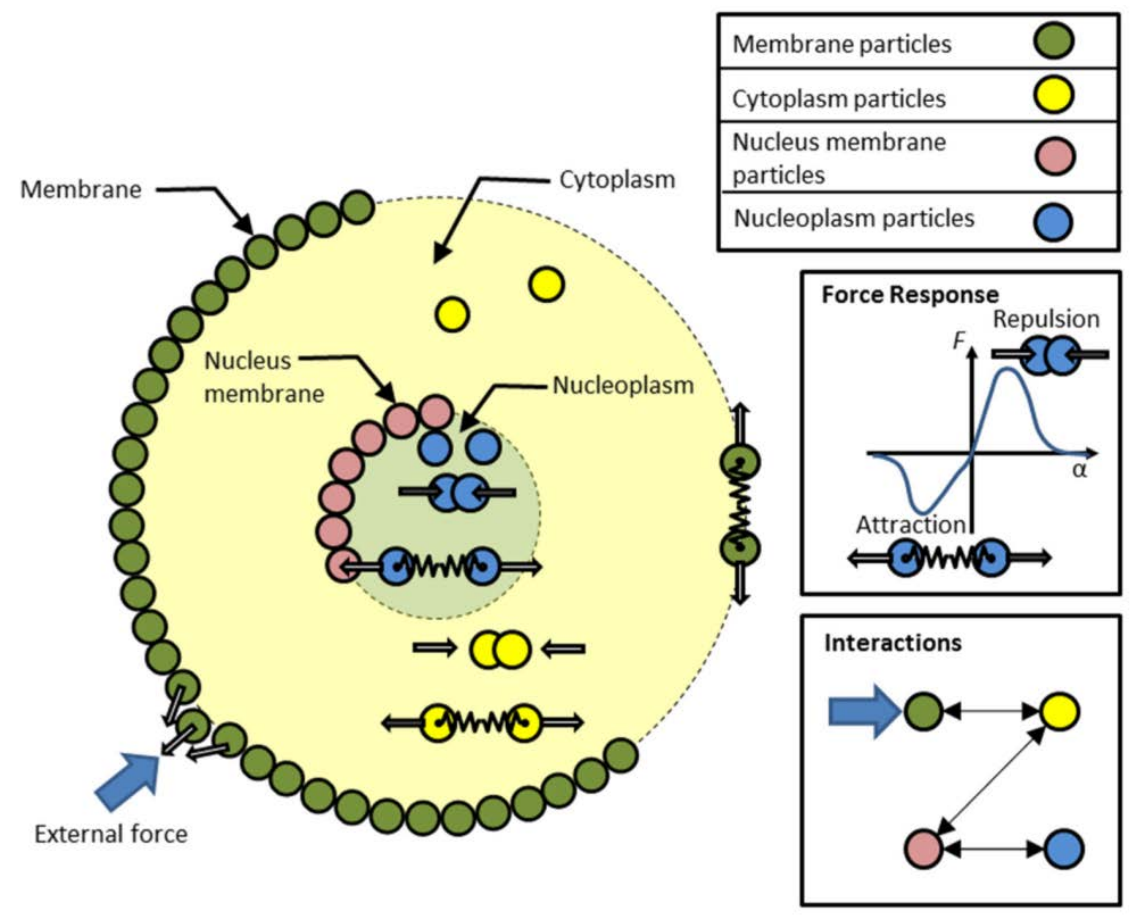

Fig. 1. The DEM model of a chondrocyte. Each cell is modelled as a collection of particles having different properties and behavior. Each particle interacts with its neighboring particles through simple interaction laws.

The mechanical behavior of each particle is governed by the interaction forces with its neighboring particles. We only consider short-range normal interaction 
forces between particles, which we model as a nonlinear spring [6]. The elastic (spring) interaction force acting on particle $I$ due to particle $J$ is defined as:

$$
\mathbf{F}_{I-J}=\left[F_{I}^{a}-P_{I-J}\left(\alpha_{I-J}\right)\right] \mathbf{n}_{I-J}
$$

where $F_{I}^{a}$ is a constant attractive force between particles of the same type and $P_{I-J}$ is a force dependent on the overlap/separation between particles, defined as the sum of particles' radii minus the distance between the particles' centers position:

$$
\alpha_{I-J}=\left(R_{I}+R_{J}\right)-\left\|\mathbf{x}_{I}-\mathbf{x}_{J}\right\|
$$

and $\mathbf{n}_{I-J}$ is the unit vector from the position of particles $I$ towards the position of particle $J$ :

$$
\mathbf{n}_{I-J}=\frac{\mathbf{x}_{J}-\mathbf{x}_{I}}{\left\|\mathbf{x}_{I}-\mathbf{x}_{J}\right\|}
$$

In the simplest form, the force $P_{I-J}$ is chosen as a linear elastic force [6]:

$$
P_{I-J}\left(\alpha_{I-J}\right)=\left\{\begin{array}{cc}
0, & -\delta_{I-J}>\alpha_{I-J} \\
s_{I-J} R_{I-J} \alpha_{I-J}, & \alpha_{I-J} \geq-\delta_{I-J}
\end{array}\right.
$$

where $S_{I-J}$ is the stiffness parameter for the spring between particles I and $\mathrm{J}$ and $R_{I-J}$ is the equivalent radius.

The elastic force is zero if particle $J$ is outside the interaction distance for particles $I$ and $J,-\delta_{I-J}>\alpha_{I-J}$ (have limited range). More complicated interactions can be included, such as non-linear normal forces, tangential forces and torques, in order to capture complex macroscopic material behavior [6]; however, given the already large variability in tissue properties and the uncertainty in determining mechanical parameters, we have not included such forces.

The equivalent radius appearing in eq. (4) is computed based on the individual radii of the two particles, and is included for scaling the response with the size of the particles:

$$
R_{I-J}=\frac{R_{I} R_{J}}{R_{I}+R_{J}}
$$

The total force acting on particle $I$ due to its neighbouring particles is obtained by summing the influences of all surrounding particles:

$$
\mathbf{F}_{I}=\sum_{J \neq I} \mathbf{F}_{I-J}
$$

Because only short range interactions are considered, the summation in eq. (6) has non-zero terms only for $n_{I}$ neighbouring particles, as resulting from eq. (4). We will name this set of neighbouring particles $N_{I}$.

The motion of particle $I$ is governed by Newtonian physics [6]:

$$
m_{I} \ddot{\mathbf{x}}_{I}=\sum_{J \in N_{I}} \mathbf{F}_{I-J}+\mathbf{F}_{I}^{E}
$$

with the externally applied force $\mathbf{F}_{I}^{E}$ including any other forces other than particle interactions (such as gravity). 
Given the very slow evolution of the growth plate, we can consider the chondrocyte behavior a very slowly evolving phenomena; therefore, the viscous interaction forces are ignored and do not appear in the above equation of motion. For the same reason, the inertial forces can also be ignored, and the mechanical system can be considered in a quasi-static equilibrium at any point in time.

\section{Quasi-static Solution Method}

The quasi-static equilibrium equation obtained by ignoring the inertial term from eq. (7) requires the solution of a very large (and possible non-linear) system of equations at each time step. We propose to use an explicit solution method, based on the addition of artificial transients to eq. (7) (dynamic relaxation), to reach the steady state solution, as described in the following sections. Such solution method allows the equations in the system of equations (7) to be decoupled and solved individually at each time step, being well suited for parallel implementation. We adopted the following procedure, inspired from the finite element $[7,8]$ and meshless $[9,10]$ methods, consisting of the inclusion of a mass proportional damping term in the equation of motion (7):

$$
m_{I} \ddot{\mathbf{x}}_{I}+c \cdot m_{I} \dot{\mathbf{x}}_{I}=\sum_{J \in N_{I}} \mathbf{F}_{I-J}+\mathbf{F}_{I}^{E}
$$

where $c$ is the damping coefficient.

The fastest convergence is obtained [7] when the damping parameter and time step values are:

$$
\begin{aligned}
c & \approx 2 \sqrt{A_{0}}=2 \omega_{0}, \\
\Delta t & \approx 2 / \sqrt{A_{m}}=2 / \omega_{\text {max }},
\end{aligned}
$$

where $A_{0}$ and $A_{m}$ are the minimum and maximum eigenvalues of matrix $\mathbf{A}=\mathbf{M}^{-1} \mathbf{K}$, $\mathbf{K}$ being the stiffness matrix and $\mathbf{M}$ the mass matrix of the structure (therefore, $\omega_{0}$ and $\omega_{\max }$ are the lowest and highest circular frequencies of the un-damped equation of motion). Given that the assembly of particles is equivalent to an assembly of bar elements, a similar procedure to the one described in [7] is used to allocate mass to each particle, which promotes fast convergence to the steady state and guarantees stability of the explicit time integration procedure.

\section{Neighbor Search Algorithm}

The computation of interactions between the particles involved in a simulation requires the neighbors of each particle to be identified at each time step. In explicit dynamic simulation this process becomes a major computational bottleneck; it is therefore important that the neighbor search algorithm is highly optimized [11].

To optimize the bin search algorithm, we perform the discretization using only similar sized particles. We then adopt a binning algorithm, which partitions the 
problem domain into equally sized bins, with the size of the bins selected in such a way that the neighbors of a given particles can be found in the bin containing the particle or in the neighboring bins. Once the particles are distributed into bins, their neighbors can be easily found by looking in the neighboring bins. The restriction on the particle size ensures that only a small number of particles are included in each bin. This algorithm has $\mathrm{O}(N)$ complexity, therefore its computation time increases linearly with the number of particles.

\section{Modelling of Special Cell-ECM Interactions}

To complete the modelling of chondrocyte migration through ECM, there are several special interactions which need to be considered apart from the simple particle to particle forces. These include: a membrane model which ensures cell integrity and prevents seepage of cell particles outside the cell [12], modelling ECM degradation by the chondrocyte and modelling secretions by the chondrocytes.

We implemented the membrane behavior by including constant attractive forces between the membrane particles, to create a constant tension in the membrane. Also, each membrane particle is aware of its neighboring membrane particles and does not interact with other particles of the same membrane, in order to avoid membrane folding onto itself. If a membrane particle is displaced from the central position between its neighbors, an elastic restoring force is applied to it. This is required in order to maintain the relative positions of the membrane particles.

ECM degradation by the chondrocyte has been implemented by introducing an integrity measure for each of the ECM particles. When an ECM particle I comes into contact with a membrane particle $J$ located on the leading edge of the chondrocyte, its integrity is reduced by a constant value (degradation factor, $D$ ) multiplied by the particle penetration (as a measure of the contact surface) at each time step:

$$
\mathrm{I}_{I}^{t+1}=\mathrm{I}_{I}^{t}-D \alpha_{I-J}, \quad \alpha_{I-J}>0
$$

When the integrity of a particle becomes negative the particle is removed from the simulation.

The forces between particles are modified by introducing the integrity measure above as:

$$
\bar{P}_{I-J}=\mathrm{I}_{I} \mathrm{I}_{J} P_{I-J}
$$

The secretions generated by the chondrocyte are modelled by increasing the size of any ECM particle $I$ which come into contact with a membrane particle $J$ located on the secreting part of the membrane. The size increase is generated by multiplying the radius of the ECM particles by a constant growth factor $G>1$ at each time step:

$$
R_{I}{ }^{t+1}=G R_{I}{ }^{t}, \quad \alpha_{I-J}>0
$$

Cell growth is modelled in a similar manner, using pre-defined growth factors for cytoplasm and nucleus particles. When the radius of the particle becomes 
higher than the maximum radius used in the simulation (imposed due to the neighbor search algorithm, which is optimized for same size particles), the particle is split into 3 equally sized particles (in 2D), whose radius is selected so that the occupied area is preserved.

\section{Simulation of Chondrocytes in the Growth Plate}

Chondrocytes in the growth plate undergo a differentiation cascade which drives the longitudinal growth of the skeletal elements through chondrocyte proliferation, cellular enlargements via hypertrophy, ECM synthesis and controlled matrix degradation [5]. The growth plate is organized into layers of resting, proliferative and hypertrophic chondrocytes. In the resting layer the chondrocytes are round and rarely divide. In the proliferative zone, the cells undergo rapid division, are flattened along the mediolateral axis, and form columns along the proximodistal axis of the long bones. Chondrocytes end their cycle at the proximal end of the column and increase their volume to become hypertrophic before the zone is calcified and replaced by trabecular bone [5].

Chondrocytes are surrounded by a thin pericellular matrix (PCM), which is enveloped by the territorial matrix (TM). The ECM around the columns is called the inter-territorial matrix (ITM) and fills up the longitudinal septum between the chondrocyte clusters. One column in the proliferative zone usually consists of several flattened chondrocytes surrounded by different matrix compartments. The PCM and TM together with the clustered chondrocytes define the columnar chondron, the functional unit of the cartilage [1], which is considered to play an important role in regulating the interactions between chondrocytes and their surrounding matrix. Investigations of the elastic properties of the proliferative zone of the murine growth plate by atomic force microscopy (AFM) have indicated that ITM has an approximately two times higher stiffness compared to TM/PCM [5].

During its life cycle, the growth plate chondrocyte secrets different molecules, ranging from randomly oriented type II collagen and hydrophilic proteoglycans in the proliferative zone to metalloproteinases (MMPs), which participate in the reabsorption of the ECM, in the hypertrophic zone [13]. We are going to use our DEM framework to study the effect of aggrecan secretion on the shape of the chondrocytes in the proliferative zone and chondrocyte enlargement, combined with ECM degradation by MMPs, in the hypertrophic zone. The particle interactions used in the simulation are described in Table 1, with the parameter values given in Table 2. The boundary conditions consisted of fixed ECM particles on the edges of the problem domain. We consider the cell to be polarized, with the secretion/ECM degradation only happening on an active portion of the cell membrane. We investigated the influence of nucleus stiffness on the cell flattening by running a simulation with the nucleus having the same stiffness as the cytoplasm. 

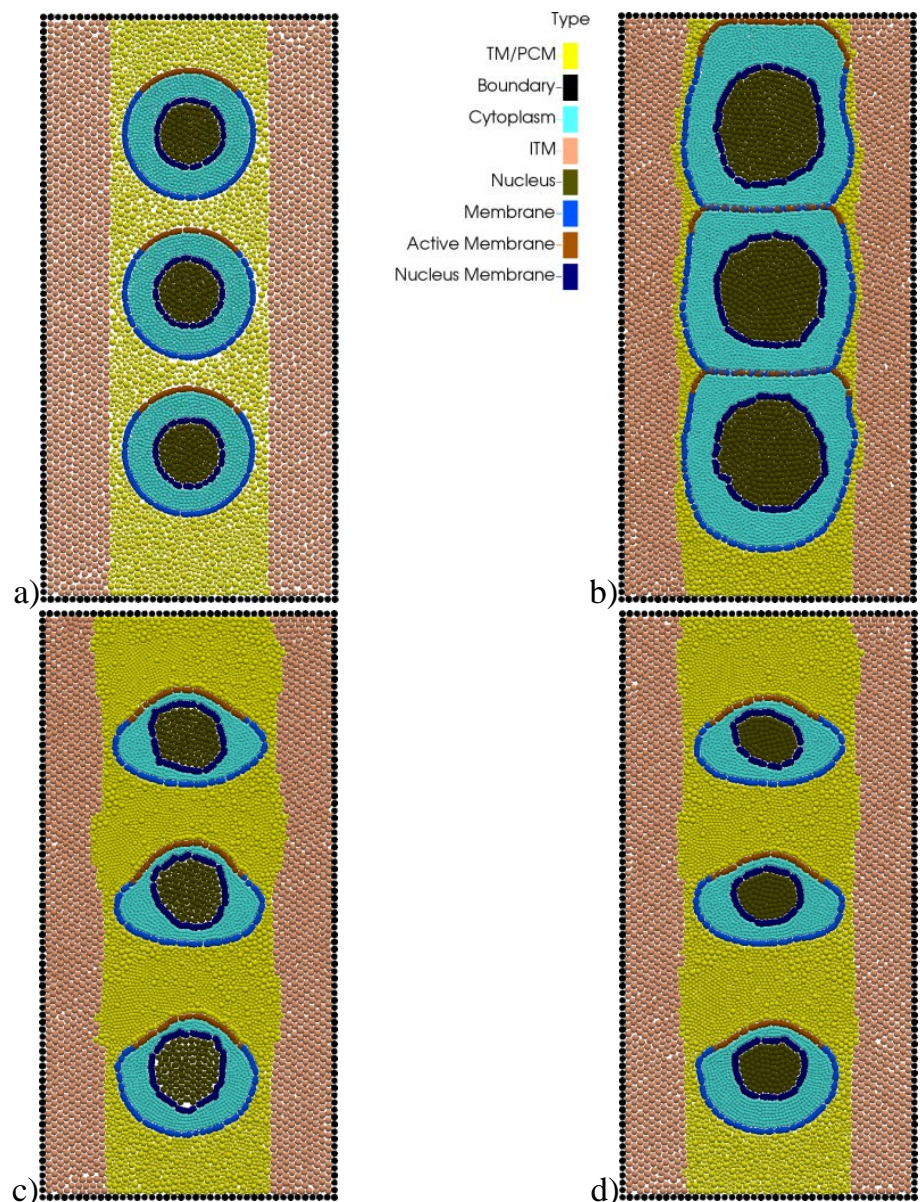

Fig. 2. Results of simulating mechanical behavior of chondrocytes in the growth plate. a) Initial configuration (7121 particles); b) Results for chondrocyte enlargement in the hypertrophic zone, with ECM degradation by MMPs at the active edge (22068 particles); c) Results for aggrecan secretion in the proliferative zone, nucleus 2.5 times stiffer than cytoplasm (16304 particles); d) Results for aggrecan secretion in the proliferative zone, nucleus same stiffness as cytoplasm (14941 particles).

Table 1. Particle types and interactions used in the simulation (the table is symmetrical).

\begin{tabular}{|l|c|c|c|c|c|c|c|}
\hline Particle Type & T & I & C & N & M & NM & AM \\
\hline TM/PCM (T) & $P_{T}$ & & & & & & \\
\hline ITM (I) & $P_{T I}$ & $P_{I}$ & & & & & \\
\hline Cytoplasm (C) & & & $P_{C}$ & & & & \\
\hline Nucleus (N) & & & & $P_{N}$ & & & \\
\hline Membrane (M) & $P_{T M}$ & $P_{I M}$ & $P_{C M}$ & & $F_{M}^{a}, P_{M}$ & & \\
\hline Nucleus membrane (NM) & & & $P_{C N M}$ & $P_{N N M}$ & & $F_{N M}^{a}, P_{N M}$ & \\
\hline Active membrane (AM) & $D, G, P_{T M} P_{I M}$ & $P_{C M}$ & & $F_{M}^{a}, P_{M}$ & & $F_{M}^{a}, P_{M}$ \\
\hline
\end{tabular}


Because this simulation belongs to a class of problems where deformation is only driven by displacements [14], the simulation results do not depend on the actual stiffness defining the elastic forces between different particles; we only have to estimate the ratios between the stiffness of different types of tissues. Therefore we considered the nucleus to be 2.5 times stiffer than the cytoplasm [2] and the ITM 2 times stiffer than the TM/PCM [5]. Elastic forces between membrane particles were chosen to prevent particle seepage through the membrane [12]. The simulation results are presented in Figure 2.

Table 2. Simulation parameters

\begin{tabular}{|l|l|}
\hline Simulation parameters & Parameter Values \\
\hline Domain size & 40 um x $80 \mathrm{um}$ \\
\hline Chondrocyte radius & $10 \mathrm{um}$ \\
\hline Particle radius & $0.30-0.52 \mathrm{um}$ \\
\hline Number of time steps & 2000 \\
\hline Elastic force between TM/PCM particles $P_{T}$ & $s_{T}=0.5 ; \delta_{T}=0.01$ \\
\hline Elastic force between ITM particles $P_{I}$ & $s_{I}=1 ; \delta_{I}=0.01$ \\
\hline Elastic force between cytoplasm particles $P_{C}$ & $s_{C}=0.2 ; \delta_{C}=0.01$ \\
\hline Elastic force between nucleus particles $P_{N}$ & $S_{N}=0.5 ; \delta_{N}=0.01$ \\
\hline Elastic force between membrane particles $P_{M}$ & $s_{M}=5 ; \delta_{M}=0$ \\
\hline Constant force between membrane particles $F_{M}^{a}$ & 0.05 \\
\hline Elastic force between membrane particles $P_{N M}$ & $S_{N M}=0.5 ; \delta_{N M}=0$ \\
\hline Constant force between membrane particles $F_{N M}^{a}$ & 0.02 \\
\hline Elastic force between T,I, C and membrane $P_{X M}$ & $s_{X M}=1 ; \delta_{X M}=0.01$ \\
\hline Elastic force between C, N and NM $P_{X N M}$ & $s_{X N M}=0.5 ; \delta_{X N M}=0.01$ \\
\hline Elastic force between TM/PCM and ITM $P_{T I}$ & $s_{T I}=2 ; \delta_{T I}=0$ \\
\hline Degradation factor $D$ & 0.05 \\
\hline Growth factor $G$ & 1.005 \\
\hline
\end{tabular}

\section{Discussion and Conclusions}

This paper presents a discrete element method for modelling cell and tissue mechanics. By adopting a discrete element modelling framework we are able to simulate complex mechanical phenomena, including large cell deformations, ECM degradation, cell growth and cell secretions; such simulations would be very difficult to perform using continuum based simulation methods. The discrete element method facilitates easy particle insertion and removal, which is needed in simulating some of these phenomena.

We apply the proposed DEM framework to the modelling of chondrocyte behavior during its different life stages in the growth plate. We modelled the secretion of ECM components in the proliferative zone and the chondrocyte enlarge- 
ment in the hypertrophic zone combined with ECM degradation by MMPs on part of the cell's membrane. The simulation produced the expected cell behavior, resulting in some flattening of the chondrocytes in the proliferative zone and in large and square-like shaped chondrocytes in the hypertrophic zone.

The cell flattening in the proliferative zone could not be completely explained by the cell secretion alone, especially for high cell aspect ratios. This may be due to complex interactions between the chondrocyte, its pericellular microenvironment and the load bearing extracellular matrix [15]; this includes vectorial production and resorption of ECM around the chondrocytes as well as rapid cell division and reorientation [1], processes that are not captured by our current model.

The simulations took approximatively $69 \mathrm{~s}$ for the chondrocyte enlargement in the hypertrophic zone and $44 \mathrm{~s}$ for the secretion in the proliferative zone on a PC having an Intel Core i7-5930K @ 3.5 GHz processor and running Windows 8, using a single threaded implementation of the explicit integration algorithm. Given its explicit nature, the algorithm is very well suited for parallel implementation on Graphics Processing Units (GPU); we expect a large speed improvement for such an implementation, based on our previous experience with explicit finite element codes [16].

The DEM framework can be extended by including other mechanical and chemical processes (such as cell division and cell activity regulation). The possibility to couple mechanical and chemical interactions happening in the growth plate will help us gain more insight into the complex phenomena governing bone growth.

\section{Acknowledgments}

This research was supported partially by the Australian Government through the Australian Research Council's Discovery Projects funding scheme (project DP160100714). The views expressed herein are those of the authors and are not necessarily those of the Australian Government or Australian Research Council. We wish to acknowledge the Raine Medical Research Foundation for funding G. R. Joldes through a Raine Priming Grant, and the Department of Health, Western Australia, for funding G. R. Joldes through a Merit Award.

\section{Bibliography}

1. Hunziker, E.B., Mechanism of longitudinal bone growth and its regulation by growth plate chondrocytes. Microscopy Research and Technique, 1994. 28(6): 505-519.

2. Gardiner, B.S., et al., Discrete element framework for modelling extracellular matrix, deformable cells and subcellular components. PLoS Computational Biology, 2015: DOI: 10.1371/journal.pcbi.1004544.

3. Alman, B.A., The role of hedgehog signalling in skeletal health and disease. Nat Rev Rheumatol, 2015. 11(9): 552-560. 
4. Gentili, C. and R. Cancedda, Cartilage and bone extracellular matrix. Current Pharmaceutical Design, 2009. 15(12): 1334-48.

5. $\quad$ Prein, C., et al., Structural and mechanical properties of the proliferative zone of the developing murine growth plate cartilage assessed by atomic force microscopy. Matrix Biology, 2016. 50: 1-15.

6. Luding, S., Introduction to discrete element methods. European Journal of Environmental and Civil Engineering, 2008. 12(7-8): 785-826.

7. Joldes, G.R., A. Wittek, and K. Miller, Computation of intra-operative brain shift using dynamic relaxation. Computer Methods in Applied Mechanics and Engineering, 2009. 198(41-44): 3313-3320.

8. Joldes, G.R., A. Wittek, and K. Miller, An adaptive Dynamic Relaxation method for solving nonlinear finite element problems. Application to brain shift estimation. International Journal for Numerical Methods in Biomedical Engineering, 2011. 27(2): 173-185.

9. Horton, A., et al., A Meshless Total Lagrangian Explicit Dynamics Algorithm for Surgical Simulation. International Journal for Numerical Methods in Biomedical Engineering, 2010. 26(8): 977-998.

10. Joldes, G.R., A. Wittek, and K. Miller, Stable time step estimates for mesh-free particle methods. International Journal for Numerical Methods in Engineering, 2012. 91(4): 450-456.

11. Williams, J.R., E. Perkins, and B. Cook, A contact algorithm for partitioning $N$ arbitrary sized objects. Engineering Computations, 2004. 21(2/3/4): 235-248.

12. Gardiner, B.S., et al., Controlling seepage in discrete particle simulations of biological systems. Computer Methods in Biomechanics and Biomedical Engineering, 2016. 19(10): 1160-1170.

13. Blair, H.C., M. Zaidi, and P.H. Schlesinger, Mechanisms balancing skeletal matrix synthesis and degradation. Biochemical Journal, 2002. 364(Pt 2): 329-341.

14. Miller, K. and J. Lu, On the prospect of patient-specific biomechanics without patient-specific properties of tissues. Journal of the Mechanical Behavior of Biomedical Materials, 2013. 27: 154-166.

15. Poole, C.A., S. Ayad, and R.T. Gilbert, Chondrons from articular cartilage. V. Immunohistochemical evaluation of type VI collagen organisation in isolated chondrons by light, confocal and electron microscopy. Journal of Cell Science, 1992. 103(4): 1101-1110.

16. Joldes, G.R., A. Wittek, and K. Miller, Real-Time Nonlinear Finite Element Computations on GPU - Application to Neurosurgical Simulation. Computer Methods in Applied Mechanics and Engineering, 2010. 199(49-52): 3305-3314. 